\title{
gs \\ Electron acceleration and generation of high-brilliance $x$-ray radiation in kilojoule, subpicosecond laser-plasma interactions
}

\author{
J. Ferri* \\ CEA, DAM, DIF, 91297 Arpajon, France and Laboratoire d'Optique Appliquée, ENSTA, \\ CNRS, Ecole Polytechnique, UMR 7639, 91761 Palaiseau, France \\ X. Davoine \\ CEA, DAM, DIF, 91297 Arpajon, France \\ S. Y. Kalmykov \\ Department of Physics and Astronomy, University of Nebraska-Lincoln, Lincoln, \\ Nebraska 68588-0299, USA \\ A. Lifschitz \\ Laboratoire d'Optique Appliquée, ENSTA, CNRS, Ecole Polytechnique, \\ UMR 7639, 91761 Palaiseau, France \\ (Received 4 May 2016; published 3 October 2016)
}

\begin{abstract}
Petawatt, picosecond laser pulses offer rich opportunities in generating synchrotron x-rays. This paper concentrates on the regimes accessible with the PETAL laser, which is a part of the Laser Megajoule (LMJ) facility. We explore two physically distinct scenarios through Particle-in-Cell simulations. The first one realizes in a dense plasma, such that the period of electron Langmuir oscillations is much shorter than the pulse duration. Hallmarks of this regime are longitudinal breakup ("self-modulation") of the picosecond-scale laser pulse and excitation of a rapidly evolving broken plasma wake. It is found that electron beams with a charge of several tens of $\mathrm{nC}$ can be obtained, with a quasi-Maxwellian energy distribution extending to a few-GeV level. In the second scenario, at lower plasma densities, the pulse is shorter than the electron plasma period. The pulse blows out plasma electrons, creating a single accelerating cavity, while injection on the density downramp creates a nC quasi-monoenergetic electron bunch within the cavity. This bunch accelerates without degradation beyond $1 \mathrm{GeV}$. The $\mathrm{x}$-ray sources in the self-modulated regime offer a high number of photons $\left(\sim 10^{12}\right)$ with the slowly decaying energy spectra extending beyond $60 \mathrm{keV}$. In turn, quasimonoenergetic character of the electron beam in the blowout regime results in the synchrotron-like spectra with the critical energy around $10 \mathrm{MeV}$ and a number of photons $>10^{9}$. Yet, much smaller source duration and transverse size increase the x-ray brilliance by more than an order of magnitude against the self-modulated case, also favoring high spatial and temporal resolution in x-ray imaging. In all explored cases, accelerated electrons emit synchrotron x-rays of high brilliance, $B>10^{20}$ photons $/ \mathrm{s} / \mathrm{mm}^{2} / \mathrm{mrad}^{2} / 0.1 \% \mathrm{BW}$. Synchrotron sources driven by picosecond kilojoule lasers may thus find an application in $\mathrm{x}$-ray diagnostics on such facilities such as the LMJ or National Ignition Facility (NIF).
\end{abstract}

DOI: 10.1103/PhysRevAccelBeams.19.101301

\section{INTRODUCTION}

Recent progress in ultrafast, high-power laser technology has spurred development of compact, all-optical laserplasma electron accelerators (LPAs) [1,2]. These accelerators, taking advantage of a $\mathrm{GV} / \mathrm{cm}$-scale accelerating gradient of a laser-driven plasma wake [3-6], are seen as a viable cost-effective alternative to the conventional solid

\footnotetext{
julien.ferri@cea.fr

Published by the American Physical Society under the terms of the Creative Commons Attribution 3.0 License. Further distribution of this work must maintain attribution to the author(s) and the published article's title, journal citation, and DOI.
}

structure-based technology [7]. Architecture of operational LPAs is remarkably diverse, from sub-mm structures driven by $\mathrm{mJ}$-scale, $\mathrm{kHz}$ repetition-rate pulses [8,9] to foot-scale designs driven by petawatt single-shot machines [10]. Generated beams range in parameters from tightly collimated, background-free, high-brightness, multi-hundred $\mathrm{MeV}$ electron beams [11-13] to broadband, high-current distributions protruding into a $\mathrm{GeV}$ range $[10,14-18]$. Impressive versatility of the LPA makes it a popular research tool for radiographic studies [19], ultrafast chemistry [20], medicine [21], and national security applications [22].

The LPA is a natural source of high-brilliance $\mathrm{x}$-rays [23]. Electrons confined in the focusing zone of the plasma 
wake experience transverse betatron oscillations, emitting highly collimated synchrotron $\mathrm{x}$-rays predominantly in the direction of their propagation $[24,25]$. Improvements in the laser technology helped extend the energy of photons from 10 to $100 \mathrm{keV}$ range and improve their collimation [25-27]. Additionally, the use of a guiding capillary can enhance the fluence of x-rays by more than one order of magnitude [28-30]. With their fs-scale duration, high spatial coherence owing to the micron-scale source size, and a brilliance of order $10^{22}$ photons $/ \mathrm{s} / \mathrm{mm}^{2} / \mathrm{mrad}^{2} / 0.1 \% \mathrm{BW}$ [26], pulsed synchrotron $\mathrm{x}$-rays from plasma wakes are a perfect fit for high-resolution phase contrast imaging [31-33]. Most of the experiments focused on this task have been conducted with sub-100 fs, Joule-energy laser pulses, which limited the electron energy to a few hundreds of $\mathrm{MeV}$ and critical photon energy to the sub- $10 \mathrm{keV}$ range. $\mathrm{X}$-rays with critical energies in tens of $\mathrm{keVs}$ have been produced with the laser pulses of energy between 0.1 and $0.3 \mathrm{~kJ}$ and duration between $0.2 \mathrm{ps}$ [10] and $0.75 \mathrm{ps}$ [34]. Application of kJ-scale, ps-length pulses, delivered by facilities operational or under construction in the national laboratories worldwide [35-39], brings the promise of further increase in photon number, energy, and brilliance of x-rays, which are essential for ultrafast phase contrast imaging, radiography, or spectroscopy of dense plasmas ubiquitous in inertial confinement fusion (ICF), laboratory astrophysics, warm dense matter, or shock experiments [40]. This paper concentrates on the regimes of electron acceleration and $\mathrm{x}$-ray production accessible with the lasers similar to PETAL [41], which is a part of the Laser Megajoule (LMJ) framework dedicated primarily to the ICF physics studies. The kJ PETAL pulse is expected to have a half-ps duration, and thus $\approx 2 \mathrm{PW}$ power. We show that these parameters of the driver permit small source size (sub- $25 \mu \mathrm{m}$ ) and short duration of the x-ray pulse (sub-250 fs). Owing to their competitive brilliance $\left(10^{20}-10^{22}\right.$ photons $/ \mathrm{s} / \mathrm{mm}^{2} / \mathrm{mrad}^{2} / 0.1 \% \mathrm{BW}$, depending on the regime), these $\mathrm{x}$-ray sources would be an asset for ultrafast diagnostics of high-density plasmas, contributing to the LMJ mission.

Dynamics of the LPA with petawatt, 100 fs-scale pulses is well understood [42] and readily lends itself to all-optical control $[43,44]$. Extending the pulse duration to the ps level brings new challenges. One of them is accessibility of the blowout regime [45-48], which has been so far the only option to produce quasimonoenergetic collimated electron beams. Blowout in the tenuous plasma $\left(n_{e} / n_{c} \ll 1\right.$, where $n_{e}$ is the background electron density, $n_{c}=\omega_{0}^{2} m_{e} \varepsilon_{0} / e^{2}$ is the critical density for the radiation with a frequency $\omega_{0}, \varepsilon_{0}$ is the vacuum permittivity, $m_{e}$ is the electron rest mass, and $e$ is the electron charge) is created by a short pulse of relativistic intensity. The focused pulse with a normalized peak vector potential $a_{0} \gtrsim 3$ (where $a_{0}=|e| A_{\perp} /\left(m_{e} c\right)$, and $c$ is the speed of light) expels all electrons on its path leaving bare ions behind. The charge separation field attracts the bulk electrons to the axis, thus creating a "bubble" of electron density encompassing the pulse, propagating at a near-luminal speed. Meanwhile, plasma nonlinearities cause continuous deformations of the optical driver, and hence of the bubble, facilitating self-injection of initially quiescent electrons [49]. The expected outcome of the acceleration process can be deduced from scaling laws [50,51], which are based on two fundamental optical processes-self-guiding, in a cavitated electron channel, of a strongly overcritical laser beam (such as $P / P_{c r} \gg 1$, where $P$ is the laser power, and $P_{c r}=16.2 n_{c} / n_{e} \mathrm{GW}$ is the critical power for relativistic self-focusing [52]), and depletion of the laser pulse driving the cavitated wake [53]. To operate in this blowout regime and benefit from its scaling laws, the laser and plasma characteristics should satisfy the matching conditions. This imposes constraints on the plasma density as well as on the laser intensity, power, waist, and duration. Our study shows that the halfps PETAL pulse fits rather well into these constraints, and the blowout can be easily reached. However, large pulse duration dictates acceleration in the low-density plasma, $n_{e} \sim 10^{16} \mathrm{~cm}^{-3}$. This rules out conventional self-injection, and other injection methods have to be used.

Blowout regime is not the only option to generate brilliant $\mathrm{x}$-rays. Increasing the plasma density far beyond the limit imposed by the blowout scaling laws can lead to longitudinal breakup ("self-modulation") of the laser pulse [54-57]. As a result, the wake takes the form of a rapidly evolving train of electron cavities driven by relativistically intense optical bullets [58,59]. This turns the electron spectrum into quasi-Maxwellian, while increasing the accelerated charge by more than an order of magnitude. We shall see that this regime ramps up the number of emitted photons by two orders of magnitude against the blowout case, while maintaining the brilliance in the range of $10^{20}$ photons $/ \mathrm{s} / \mathrm{mm}^{2} / \mathrm{mrad}^{2} / 0.1 \% \mathrm{BW}$.

The paper is organized as follows. Section II specifies the numerical setup for the particle-in-cell (PIC) simulations carried out using the quasi-cylindrical code CALDER-CIRC [60]. For the parameters accessible with the PETAL laser ( $\mathrm{kJ}$ pulse energy, half-ps duration), we explore the trends in self-modulated laser wakefield acceleration (SM-LWFA) offered by variation of the background plasma density. In the high-density range $\left(n_{e}>10^{18} \mathrm{~cm}^{-3}\right)$, quasiMaxwellian electron distributions with temperatures above $100 \mathrm{MeV}$ extend to $\sim 2.5 \mathrm{GeV}$, while containing over a $\mathrm{nC}$ charge in the range above $1 \mathrm{GeV}$ (total charge 30-50 nC). Section III concentrates on acceleration in the blowout regime. At the lowest density limit prescribed by scalings, the pulse is marginally overcritical, $P / P_{c r} \approx 3.4$. Simulations carried out in this regime show that the pulse diffracts early, and does not maintain electron cavitation all the way through electron dephasing. Acceleration is thus not as efficient as predicted by scalings. Yet a $\mathrm{nC}$ electron beam is accelerated to $1.6 \mathrm{GeV}$ without accumulation of 
background, while having $<10 \%$ energy spread. Section IV explores $\mathrm{x}$-ray production. In the SM-LWFA regime, the source size and duration reveal very weak sensitivity to the background density, staying at the $25 \mu \mathrm{m}$ and $250 \mathrm{fs}$ levels, respectively. Nevertheless, extremely high charge accelerated at high densities $\left(n_{e}>10^{18} \mathrm{~cm}^{-3}\right)$ yields the brilliance close to $6 \times 10^{20}$ photons $/ \mathrm{s} / \mathrm{mm}^{2} / \mathrm{mrad}^{2} / 0.1 \% \mathrm{BW}$ (at $10 \mathrm{keV}$ ), with the estimated critical energy $\sim 26 \mathrm{keV}$. In the optimal case, the $x$-ray beam of $\approx 260$ fs duration contains $8.1 \times 10^{11}$ photons between $2 \mathrm{keV}$ and $60 \mathrm{keV}$ (total photon energy $1.5 \mathrm{~mJ}$ ). Operating in the blowout regime reduces the $\mathrm{x}$-ray photon yield by a factor 400 . Yet, the reduced source size $(7 \mu \mathrm{m})$ and duration (20 fs) ramp up the brilliance to nearly $4 \times 10^{22}$ photons $/ \mathrm{s} / \mathrm{mm}^{2} / \mathrm{mrad}^{2} / 0.1 \% \mathrm{BW}$ at $10 \mathrm{keV}$. We conclude that the promising parameters of plasma-based, all-optical synchrotron x-ray sources, driven by the kJ, half-ps laser pulses, are encouraging for their use in radiography studies and ultrafast diagnostics of dense plasmas at facilities dedicated to the ICF and high energy density science (such as NIF and LMJ).

\section{ELECTRON ACCELERATION IN THE SELF-MODULATED REGIME}

We consider interaction between a circularly polarized Gaussian laser pulse and a fully-ionized gas jet. The pulse parameters match those expected for the PETAL laser [41]. Notably, the laser pulse energy (1.1 kJ) is at least an order-ofmagnitude larger than in the previous studies of synchrotron sources in the self-modulation and blowout regimes [34,59]. This section reports the case studies corresponding to the laser power $P=2.2 \mathrm{PW}$, the pulse full width at half-maximum (FWHM) in intensity $\tau_{0}=0.5 \mathrm{ps}$, wavelength $\lambda_{0}=1 \mu \mathrm{m}$, and the waist radius at $\exp (-2)$ of peak intensity $w_{0}=42 \mu \mathrm{m}$. This yields the peak normalized vector potential $a_{0}=7.5$. The simulations are carried out with the quasicylindrical PIC code CALDER-CIRC [60]. To ensure correct laser pulse propagation, we set the time step $\Delta_{t}=0.124 \omega_{0}^{-1}$, and longitudinal and radial cell sizes respectively $\Delta_{x}=0.125 c / \omega_{0} \approx \lambda_{0} / 50$ and $\Delta_{r}=4 c / \omega_{0}$ with 12 particles per cylindrical cell. A third-order interpolation is used for the macroparticles. Three Fourier modes have been used in decomposition of currents and electric and magnetic fields. Besides, the magnetic field is interpolated quadratically in time to improve accuracy of calculation of the transverse forces. The large pulse length and the need for a fine spatial and temporal resolution incur heavy computational costs, which are only marginally offset by using a moving window. This limits our ability to carry out expansive parametric studies, yet permits us to evaluate the trends in $\mathrm{x}$-ray source parameters brought about by the variation of plasma density.

To experience the longitudinal breakup ("selfmodulation"), the laser pulse must be much longer than the period of Langmuir electron oscillations, $c \tau_{0} \gg \lambda_{p}=2 \pi c / \omega_{p}$, where $\omega_{p}=\sqrt{n_{e} e^{2} / m_{e} \varepsilon_{0}}$ and $c \tau_{0}=150 \mu \mathrm{m}$. In the simulations, the plasma begins with a $1 \mathrm{~mm}$-long linear ramp followed by a plateau. Four runs have been carried out with the density in the plateau area changing between $n_{e}=2.8 \times 10^{17} \mathrm{~cm}^{-3} \quad\left(\lambda_{p} \approx 63 \mu \mathrm{m}\right) \quad$ and $n_{e}=2.8 \times$ $10^{18} \mathrm{~cm}^{-3}\left(\lambda_{p} \approx 20 \mu \mathrm{m}\right)$. Due to the high computational cost, all four runs were terminated at $4 \mathrm{~cm}$ of pulse propagation.

Figures 1(a)-1(d) show snapshots of the laser envelope at different times for a plasma density $n_{e}=1.1 \times 10^{18} \mathrm{~cm}^{-3}$. The laser rapidly self-focuses, reaching the maximum amplitude $a_{0} \approx 19$ after $3.9 \mathrm{~mm}$ of propagation $(70 \%$ of the Rayleigh length). At this point, it starts driving a highamplitude near-resonant wakefield. Three-dimensional buckets of electron density, acting as comoving lenses, focus and defocus laser radiation. As a result, the laser beam gradually breaks up into several beamlets with a characteristic size close to the plasma wavelength, $\lambda_{p} \approx 30 \mu \mathrm{m}$. After one centimeter of propagation (approximately two Rayleigh lengths), the pulse turns into a train of four distinct beamlets, each residing in a zone of decelerating field for electrons [compare Figs. 1(d) and 1(h)]. Negative longitudinal electric field in the regions following each beamlet exceeds the cold wavebreaking limit $E_{0}=m_{e} c \omega_{p} / e$. This results in trapping and accelerating the electrons, as is clearly seen in the snapshots of the longitudinal phase space in Figs. 1(i)-1(1). However, the coupling between laser and wakefield leads to a time-evolving wakefield structure. The positions of the accelerating field regions are not stable over a long distance. The resulting phase mixing of electrons in accelerating buckets causes rapid maxwellization of electron energy spectrum.

Accumulation of the trapped charge in the course of laser pulse propagation is characterized in Fig. 2(a). The total charge of electrons accelerated beyond $70 \mathrm{MeV}$ is shown as a function of propagation distance for different plasma densities. Increase in the background density increases the charge, up to $50 \mathrm{nC}$ after $1.5 \mathrm{~cm}$ of propagation in the highest-density plasma $\left(n_{e}=2.8 \times 10^{18} \mathrm{~cm}^{-3}\right)$. At this point, this high-density plasma almost fully depletes the pulse, and $Q$ starts to decrease. Steady reduction in the charge with distance is mostly due to radial expulsion of electrons from the wakefield, rather than due to their energy loss. Electron density $n_{e}=1.1 \times 10^{18} \mathrm{~cm}^{-3}$ appears to be optimal: even though the charge accumulation stops after $2 \mathrm{~cm}$ at $Q \approx 40 \mathrm{nC}$, subsequent reduction is insignificant. For the lowest densities explored here, $n_{e}=5.6 \times$ $10^{17} \mathrm{~cm}^{-3}$ and $n_{e}=2.8 \times 10^{17} \mathrm{~cm}^{-3}$, the injection and acceleration process carry on after $4 \mathrm{~cm}$ of propagation.

The quasi-Maxwellian electron spectra after $2 \mathrm{~cm}$ of propagation are shown in Fig. 2(b). The temperature, calculated for electrons with the energy above $200 \mathrm{MeV}$, increases with the density (from $110 \mathrm{MeV}$ at $n_{e}=2.8 \times$ $10^{17}$ to $200 \mathrm{MeV}$ at $n_{e}=2.8 \times 10^{18}$ ). Note that for the highest density explored here, the charge above $1 \mathrm{GeV}$ is 

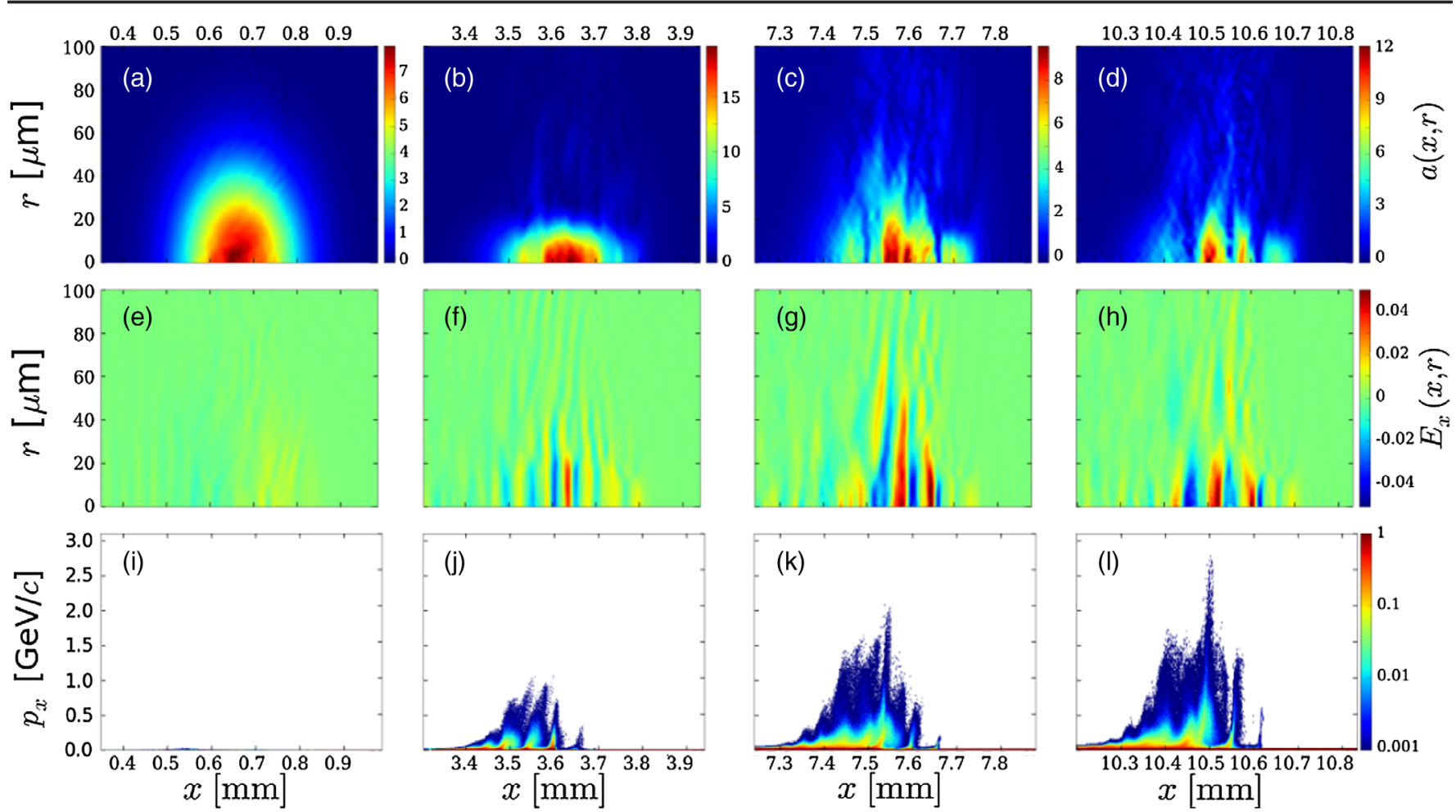

FIG. 1. Hot electron generation in the SM-LWFA at the plasma density $n_{e}=1.1 \times 10^{18} \mathrm{~cm}^{-3}$. The columns (from left to right) correspond to the propagation distances $0.9,3.9,7.9$, and $10.8 \mathrm{~mm}$ (the Rayleigh length being $5.5 \mathrm{~mm}$ ). Snapshots of the laser envelope (a-d) reveal longitudinal breakup of the pulse into beamlets with the size close to the plasma wavelength, $\lambda_{p} \approx 30 \mu \mathrm{m}$. Images (e-h) of the longitudinal accelerating field (in units $m_{e} c \omega_{0} / e$ ) reveal excitation of a broken plasma wake, which traps and accelerates electrons to $\mathrm{GeV}$ energies, as evidenced by the snapshots of longitudinal phase space (i-1).
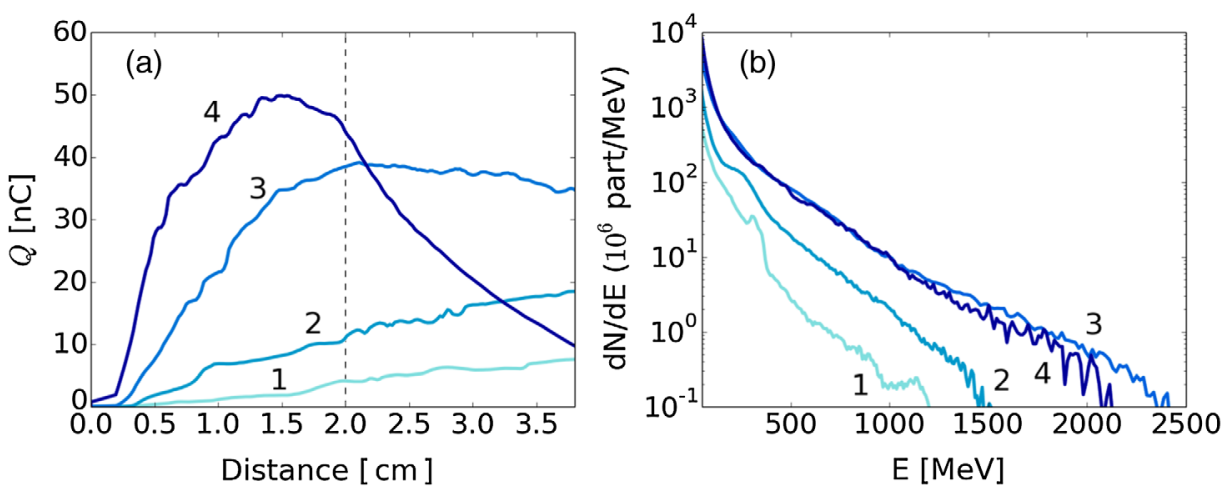

FIG. 2. Accumulation of electron charge and generation of thermal energy distributions in the SM-LWFA. (a) Electron charge $Q$ in the energy range above $70 \mathrm{MeV}$ as a function of the propagation distance. (b) Electron energy distribution after $2 \mathrm{~cm}$ of propagation. From lighter to darker blue: (1) $n_{e}=2.8 \times 10^{17} \mathrm{~cm}^{-3}$, (2) $n_{e}=5.6 \times 10^{17} \mathrm{~cm}^{-3}$, (3) $n_{e}=1.1 \times 10^{18} \mathrm{~cm}^{-3}$ and (4) $n_{e}=2.8 \times 10^{18} \mathrm{~cm}^{-3}$.

very significant $(>1 \mathrm{nC})$. For the lowest density case, a trace of a quasimonoenergetic beam can be observed around $300 \mathrm{MeV}$ on top of the Maxwellian distribution. At this density, the laser pulse spans only a pair of wakefield buckets, which stabilizes the pulse dynamics and slows down the wake evolution. Further reduction in density results in transition to the blowout regime. This remarkably improves the system stability and drastically changes the electron acceleration dynamics. This is the subject of the next section.

\section{ELECTRON ACCELERATION IN THE BLOWOUT REGIME}

First, we discuss the implications of scaling laws [51] and the prospects to carry out acceleration in the blowout regime with a PETAL-like laser. Even though the scalings do not assume any specific shape of the pulse, we consider a Gaussian pulse with the intensity distribution

$I(\zeta, r)=I_{\max } \exp \left(-4 \ln 2 \zeta^{2} /\left(c \tau_{0}\right)^{2}\right) \exp \left(-2 r^{2} / w_{0}^{2}\right)$ 


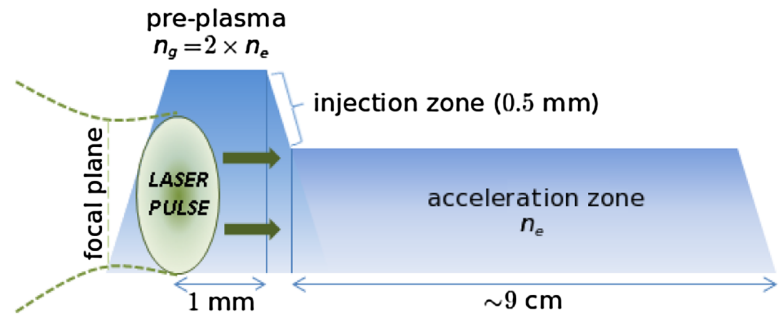

FIG. 3. Sketch of the plasma profile used in the blowout simulations, with $n_{e}=2.5 \times 10^{-5} n_{c}=2.8 \times 10^{16} \mathrm{~cm}^{-3}$.

where $\zeta=x-c t$ is a comoving Galilean variable, $r^{2}=y^{2}+z^{2}$, and $\tau_{0}$ is the pulse FWHM. $I_{\max }$ defines the peak normalized vector potential, $a_{0}=$ $0.85\left(I_{\max }\left[10^{18} \mathrm{~W} / \mathrm{cm}^{2}\right]\right)^{1 / 2} \lambda_{0}[\mu \mathrm{m}]$. For this Gaussian pulse, the peak power, peak intensity, energy, pulse duration, and the spot size are related as $P=$ $(4 \ln 2 / \pi)^{1 / 2} E / \tau_{0}=\left(\pi w_{0}^{2} / 2\right) I_{\max }$. This yields a generic engineering formula for the pulse energy,

$$
E[\mathrm{~kJ}]=2.3 \times 10^{-5} \tau_{0}[\mathrm{ps}]\left(w_{0} / \lambda_{0}\right)^{2} a_{0}^{2} .
$$

The pulse is expected to self-guide in a stable fashion, while maintaining electron cavitation. To this end, its amplitude and spot size should be matched [51], $w_{m}=2 \sqrt{a_{m}}\left(c / \omega_{p}\right)$, where $a_{m} \approx 2\left(P / P_{c r}\right)^{1 / 3} \gg 1$. We assume that the initial conditions are matched $\left(a_{0}=a_{m}, \quad w_{0}=w_{m}\right)$, and that $a_{m} \gtrsim 3$ [such that $\left.P / P_{c r}=\left(a_{m} / 2\right)^{3} \gtrsim 3.4\right]$, which is desirable to maintain electron cavitation. Then, denoting $\tau_{0}=\kappa w_{m} / c$, we express the energy of the matched pulse through its duration and wavelength:

$$
E[\mathrm{~kJ}]=\frac{2 a_{m}^{2}}{\kappa^{2}} \frac{\tau_{0}^{3}[\mathrm{ps}]}{\lambda_{0}^{2}[\mu \mathrm{m}]} \gtrsim \frac{18 \tau_{0}^{3}[\mathrm{ps}]}{\kappa^{2} \lambda_{0}^{2}[\mu \mathrm{m}]} .
$$



Using the pulse energy most effectively, that is, depleting the pulse at the moment of electron dephasing [51], requires $\kappa \approx 2 / 3$. Besides, the reduced pulse duration increases the ponderomotive force, improving electron cavitation, increasing accelerating and focusing gradients, and thus increasing probability of self-injection. However, with $\kappa \approx 2 / 3$, equation (3) yields $E=5 \mathrm{~kJ}$, which is beyond the PETAL capabilities. In turn, the expected PETAL parameters $\left(\tau_{0}=0.5 \mathrm{ps}, \lambda_{0}=1 \mu \mathrm{m}\right.$, and $\left.E=1.1 \mathrm{~kJ}\right)$ yield $\kappa \approx 1.4$ with $a_{m}=3$ or $\kappa \approx 1.9$ with $a_{m}=4$ as two possible solutions, which delimit the operating range. Using higher $\kappa$ can lead to longitudinal breakup of the pulse, and hence, to the SM-LWFA regime The lower $a_{m}$ corresponds to transition from the blowout regime to a nonlinear regime without full cavitation. With $a_{m}=3$, other matched laser and plasma parameters are $n_{e}=2.8 \times 10^{16} \mathrm{~cm}^{-3}$ and $w_{m}=110 \mu \mathrm{m}$. If we assume that electron energy in this regime is limited by dephasing [51], which occurs over a distance $L_{d}=(2 / 3)\left(n_{c} / n_{e}\right) w_{m} \simeq 3 \mathrm{~m}$, we may expect the energy gain $E_{\text {fin }} \simeq \sqrt{a_{m}}\left(\omega_{p} / c\right)\left(m_{e} c^{2} / 2\right) L_{d} \simeq 40 \mathrm{GeV}$. We shall see later that this estimate is somewhat optimistic.

Running a CALDER-CIRC simulation with the found above density, $n_{e}=2.8 \times 10^{16} \mathrm{~cm}^{-3}$, and slightly mismatched $a_{0}=4$ and $w_{0}=80 \mu \mathrm{m}$ (the Rayleigh length $2 \mathrm{~cm}$ ) reveals an opportunity of reaching beyond $\mathrm{GeV}$ in a sub-10 cm plasma, without degradation of the quasimonoenergetic beam. Numerical aspects of the simulation are the same as in Sec. II, except that two Fourier modes (instead of three) are used in decomposition of fields and current. Too slow variation of the structure (on a cm-scale length) and ultrarelativistic phase velocity of the wake [roughly corresponding to the Lorentz factor associated with the pulse group velocity, $\gamma_{g}=\left(n_{c} / n_{e}\right)^{1 / 2} \approx 200$ ] rule out conventional self-injection in a uniform plasma [49]. This makes the structure dark-current-proof. In addition,

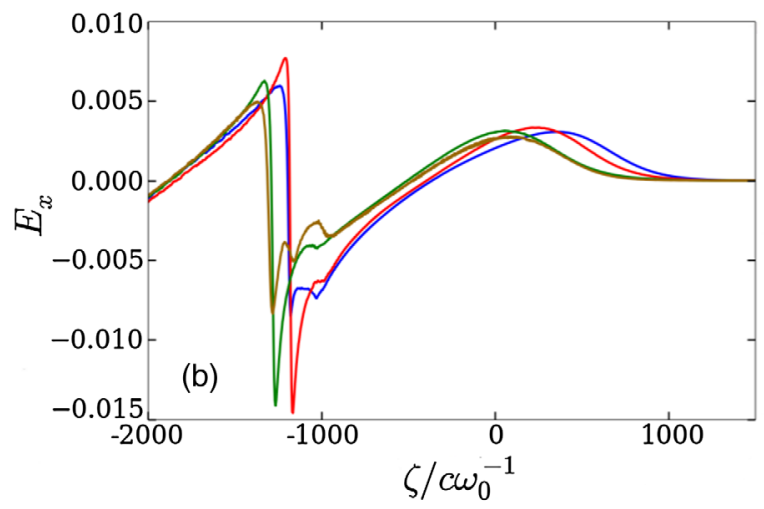

FIG. 4. Long-term pulse and wake evolution in the blowout regime. (a) WAKE simulation: evolution of the normalized peak vector potential of the pulse over $22 \mathrm{~cm}$ for the parameters used in the CALDER-CIRC simulation (the reference case: $a_{0}=4$, $n_{e}=2.8 \times 10^{16} \mathrm{~cm}^{-3}$ - solid black line), and for matched parameters corresponding to $a_{0}=4$ and $n_{e}=6.6 \times 10^{16} \mathrm{~cm}^{-3}($ dashed red line), $a_{0}=3.5$ and $n_{e}=4.4 \times 10^{16} \mathrm{~cm}^{-3}$ (dashed green line) and $a_{0}=3.18$ and $n_{e}=3.3 \times 10^{16} \mathrm{~cm}^{-3}$ (dashed blue line). (b) Accelerating field $E_{x}$ (in units $m_{e} c \omega_{0} / e$ ) on the propagation axis after $1.5 \mathrm{~cm}$ (blue), $4 \mathrm{~cm}$ (red), $6.5 \mathrm{~cm}$ (green) and $9 \mathrm{~cm}(\mathrm{gold})$ in the reference case (CALDER-CIRC simulation). 


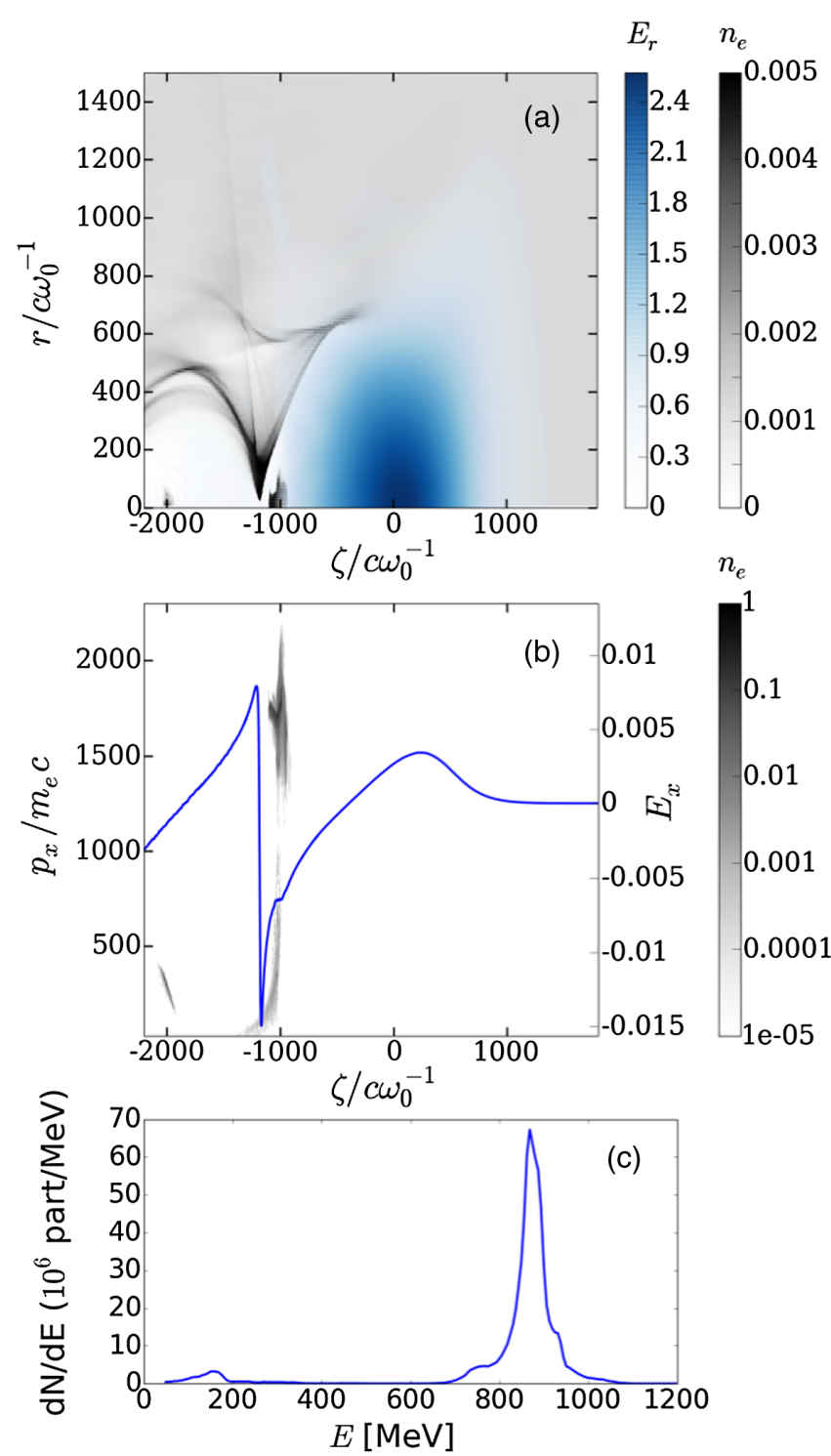

FIG. 5. Monoenergetic electron acceleration in the blowout regime. All quantities are shown at $x=4 \mathrm{~cm}$ (two Rayleigh lengths). (a) Snapshot of electron density (in units of $n_{c}$, gray), and the laser field (in units of $m_{e} c \omega_{0} / e$, blue). (b) The longitudinal field $E_{x}$ on axis (in units $m_{e} c \omega_{0} / e$, blue) and normalized density of electrons in the longitudinal phase space (gray). (c) Electron energy spectrum.

controlled injection is needed. We resort to injection in a density downramp [61,62], with the longitudinal density profile shown in Fig. 3. The density drops from $n_{g}=2 n_{e}$ to $n_{e}$ over a half-mm length, causing an expansion of the bubble sufficient to inject almost $1 \mathrm{nC}$ charge. No further injection is observed after the gradient. The lensing effect of the mm-length dense segment $[43,44]$ is obviously too weak to perturb the pulse evolution and incur additional unwanted injection in the low-density accelerator section.

The simulations show that the power ratio $P / P_{c r} \approx 3.4$ is not sufficiently high to maintain blowout through

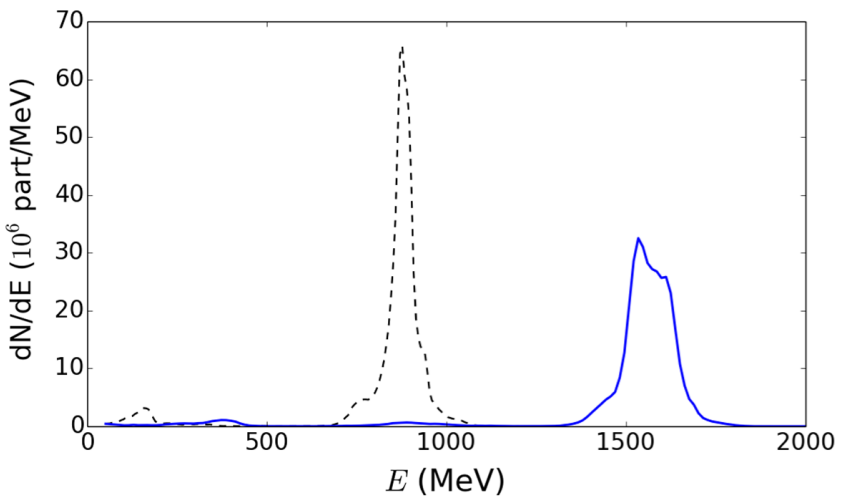

FIG. 6. Evolution of electron energy spectra in the blowout regime. Electron distribution after $9 \mathrm{~cm}$ of propagation (blue) and $4 \mathrm{~cm}$ (black dashed line).

electron dephasing, similarly to the situation explored by Kalmykov et al. [43]. Propagation over a few tens of centimeters ( $\sim 11$ Rayleigh lengths), simulated with the code WAKE [63], reveals gradual drop in the pulse intensity. Figure 4(a) shows that, in the reference case $\left(a_{0}=4, w_{0}=80 \mu \mathrm{m}\right)$, the normalized peak vector potential drops by a factor close to two after the first 8 Rayleigh lengths, which indicates a transition from the blowout to the conventional nonlinear wakefield acceleration. Gradual reduction in the accelerating gradient at the position of the electron bunch, displayed in Fig. 4(b) (from $20 \mathrm{GeV} / \mathrm{m}$ at $1.5 \mathrm{~cm}$ to $11 \mathrm{GeV} / \mathrm{m}$ at $9 \mathrm{~cm}$ ), reduces the energy gain compared with the predictions of the scaling laws. Notably, WAKE simulations with better matched parameters reveal better self-focusing and self-guiding of the pulse. Yet, propagation at higher plasma densities (hence, higher $P / P_{c r}$ ) is prone to relativistic filamentation, and is thus very demanding as regards the quality of laser beam focusing [10].

CALDER-CIRC simulation has run through $9 \mathrm{~cm}$. Snapshot of the electron density and the laser field after a $4 \mathrm{~cm}$ distance is shown in Fig. 5(a). Here, $\zeta=0$ indicates the center of the laser pulse. This is permissible as the pulse group velocity in this low density plasma is very close to $c$. Electrons have been injected primarily into the first bucket over the density gradient, with a total charge of $820 \mathrm{pC}$ above $50 \mathrm{MeV}$. At $x=4 \mathrm{~cm}$, the electron bunch has reached $0.9 \mathrm{GeV}$ [cf. Figs. 5(b) and 5(c)], and is still located at the bubble rear. This is quite natural, as this propagation distance is merely $1.3 \%$ of the dephasing length. Even though signatures of beam loading are clearly seen in Fig. 4(b), this effect does not damp significantly the accelerating field, which reaches $E_{x} \approx 20 \mathrm{GeV} / \mathrm{m}$ at the bunch location. In the end, the most energetic electrons get the energy close to $1.6 \mathrm{GeV}$ (Fig. 6), which corroborates the reduction in accelerating field caused by the diffraction of the driver. The energy spread of the electron bunch has been deteriorated, from $\Delta E_{\mathrm{FWHM}}=5.7 \%$ at $4 \mathrm{~cm}$ to $8.9 \%$ at $9 \mathrm{~cm}$. 
Finally, parameter scans with WAKE simulations reveal that operation with the 2.2 PW PETAL pulse at higher densities (up to $n_{e}=6.6 \times 10^{16} \mathrm{~cm}^{-3}$ corresponding to the borderline of the self-modulation regime) results in stronger self-focusing, guiding over a longer distance [Fig. 4(a)] and higher expected energy gain. Prospects of operation in these regimes are presently under investigation, with the results to be reported in a separate publication.

\section{SYNCHROTRON RADIATION}

Synchrotron radiation is calculated via post-processing, by plugging the trajectories of accelerated electrons into the equation [64] for the radiated energy $\frac{d^{2} I}{d \omega d \Omega}$ per frequency unit $d \omega$ and per solid angle $d \Omega$ :

$$
\begin{aligned}
\frac{d^{2} I}{d \omega d \Omega}= & \frac{1}{4 \pi \epsilon_{0}} \frac{e^{2}}{4 \pi^{2} c} \\
& \times\left|\int_{-\infty}^{\infty} \frac{\mathbf{n} \times\{[\mathbf{n}-\boldsymbol{\beta}(\dot{t})] \times \boldsymbol{\beta}(t)\}}{[1-\boldsymbol{\beta}(t) \cdot \mathbf{n}]^{2}} e^{i \omega(t-\mathbf{n} \cdot \mathbf{r}(t) / c)} d t\right|^{2} .
\end{aligned}
$$

Here, $\mathbf{r}(t)$ is the position of the electron, $\boldsymbol{\beta}(t)=\frac{\mathbf{v}(t)}{c}$ is the normalized velocity, $\dot{\boldsymbol{\beta}}(t)$ is the normalized acceleration, $\mathbf{n}$ is the direction of observation, and $\omega$ is the frequency of emitted radiation.

For simulations in the SM-LWFA regime, the x-ray output has been calculated from the trajectories of the accelerated electrons over $4 \mathrm{~cm}$ of propagation. The critical energy for betatron radiation is given by $\hbar \omega_{c}[\mathrm{eV}]=5.24 \times 10^{-21} \gamma^{2} n_{e}\left[\mathrm{~cm}^{-3}\right] r_{\beta}$, where a sinusoidal trajectory of amplitude $r_{\beta}$ is assumed [23]. Applying this to $50 \mathrm{MeV}$ electrons and $r_{\beta}=20 \mu \mathrm{m}$, we find a critical energy ranging from $0.2 \mathrm{keV}$ to $2 \mathrm{keV}$ depending on the density used. Radiation emitted by less energetic electrons has a negligible effect on the $\mathrm{x}$-ray spectrum beyond this range. We are primarily interested in the multi-keV photons, and calculate the x-ray emission only for the most energetic particles (i.e., $E>50 \mathrm{MeV}$ ), which also offsets the

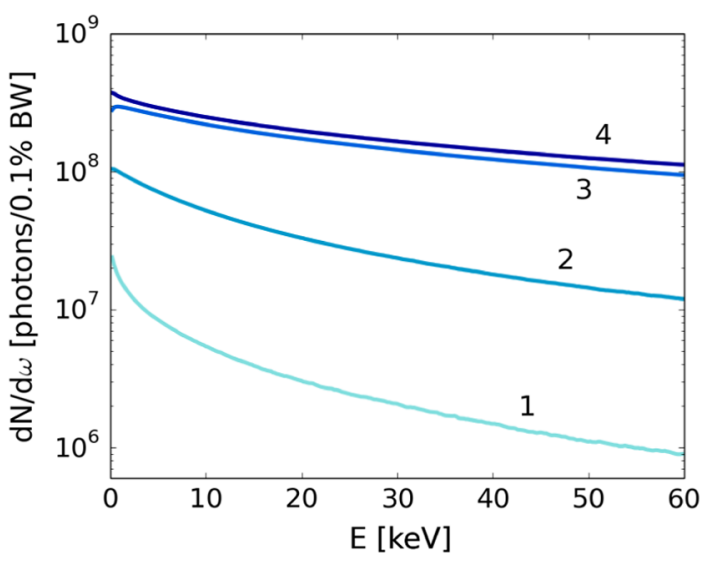

FIG. 7. Photon distribution per $0.1 \% \mathrm{BW}$ integrated over a $100 \times 100 \mathrm{mrad}^{2}$ angle, obtained in the SM-LWFA regime. From lighter to darker blue: (1) $n_{e}=2.8 \times 10^{17} \mathrm{~cm}^{-3}$, (2) $n_{e}=5.6 \times 10^{17} \mathrm{~cm}^{-3}$, (3) $n_{e}=1.1 \times 10^{18} \mathrm{~cm}^{-3}$ and (4) $n_{e}=2.8 \times 10^{18} \mathrm{~cm}^{-3}$.

computational cost. Figure 7 displays the photon distributions for the densities considered in Sec. II. The emission is broadband, with a significant spectral amplitude through several tens of keVs. Due to numerical constraints, the spectrum beyond $60 \mathrm{keV}$ has not been calculated. The total number of photons and the spectrum temperature are the highest at the highest density. This is explained by the higher accelerated charge and higher electron energy. Laser pulse rapidly depletes for $n_{e}>1.1 \times 10^{18} \mathrm{~cm}^{-3}$, unable to drive the high-amplitude wake. As a result, the x-ray yield saturates around $n_{e}=1.1 \times 10^{18} \mathrm{~cm}^{-3}$. The $x$-ray source characteristics are summed up in Table I. The number of photons within the interval 2-60 keV increases by a factor $\sim 40$ between $n_{e}=2.8 \times$ $10^{17} \mathrm{~cm}^{-3}$ and $n_{e}=2.8 \times 10^{18} \mathrm{~cm}^{-3}$, while the total radiated energy increases by a factor $\sim 60$. As the acceleration process is not finished in the lower-density cases, using longer plasmas would increase the photons number and average energy.

The increase in the number of photons with the plasma density goes along with an increase in the $\mathrm{x}$-ray divergence

TABLE I. Summary of the X-ray source parameters for the SM-LWFA (plasma lengths $4 \mathrm{~cm}$ ) and the blowout regimes (plasma length $9 \mathrm{~cm}$ ). $Q_{\max }$ is the maximal electron charge above $70 \mathrm{MeV}$ contained in the wakefield during the simulation. $N_{\gamma}$ is the number of photons emitted on the interval 2-60 keV and in an angle $100 \times 100 \mathrm{mrad}^{2}$. $\Theta_{\mathrm{FWHM}}$ is the FWHM angle of emission (mrad) and $E_{\gamma}$ the total energy of these photons. $L_{\mathrm{rms}}$ is the transverse rms size of the source and $D_{\mathrm{rms}}$ its rms duration. $B$ is the brilliance at $10 \mathrm{keV}$ (photons $/ \mathrm{s} / \mathrm{mm}^{2} / \mathrm{mrad}^{2} / 0.1 \% \mathrm{BW}$ ).

\begin{tabular}{lcccccccc}
\hline \hline$n_{e}\left(\mathrm{~cm}^{-3}\right)$ & regime & $Q_{\max }(\mathrm{nC})$ & $N_{\gamma}$ & $\Theta_{\mathrm{FWHM}}$ & $L_{\mathrm{rms}}(\mu \mathrm{m})$ & $D_{\mathrm{rms}}(\mathrm{fs})$ & $E_{\gamma}(\mathrm{mJ})$ & $B$ \\
\hline $2.8 \times 10^{16}$ & blowout & 0.82 & $2.2 \times 10^{9}$ & 4 & 7.1 & 20 & 0.003 & $3.6 \times 10^{22}$ \\
$2.8 \times 10^{17}$ & SM-LWFA & 6.9 & $2.0 \times 10^{10}$ & 18 & 25.3 & 255 & 0.03 & $1.0 \times 10^{20}$ \\
$5.6 \times 10^{17}$ & SM-LWFA & 18.2 & $1.7 \times 10^{11}$ & 42 & 25.6 & 257 & 0.31 & $1.7 \times 10^{20}$ \\
$1.1 \times 10^{18}$ & SM-LWFA & 38.7 & $7.1 \times 10^{11}$ & 47 & 25.7 & 257 & 1.52 & $5.6 \times 10^{20}$ \\
$2.8 \times 10^{18}$ & SM-LWFA & 49.8 & $8.1 \times 10^{11}$ & 75 & 29.0 & 291 & 1.76 & $1.8 \times 10^{20}$ \\
\hline \hline
\end{tabular}





FIG. 8. Angular energy distribution $\frac{d I}{d \Omega}(\mathrm{J} / \mathrm{sr})$ of the synchrotron source in the SM-LWFA regime. (a) $n_{e}=2.8 \times 10^{17} \mathrm{~cm}^{-3}$, (b) $n_{e}=5.6 \times 10^{17} \mathrm{~cm}^{-3}$, (c) $n_{e}=1.1 \times 10^{18} \mathrm{~cm}^{-3}$ and (d) $n_{e}=2.8 \times 10^{18} \mathrm{~cm}^{-3}$.

(Fig. 8). The strength parameter of the betatron oscillation scales as $K \propto r_{\beta} k_{p} \sqrt{\gamma}$, where $r_{\beta}$ is the oscillation radius. Simulations show that $r_{\beta}$ is almost independent of density (the transverse size of the X-ray source $L_{\mathrm{rms}} \sim r_{\beta}$, shown in Table I, is estimated as the rms transverse size of the electron bunch). Hence $K \propto \sqrt{n_{e} \gamma}$. As an increase in the density leads to an increase in the electron energy, the angle of emission, which is proportional to $K$, also increases. As the transverse source size and source duration weakly depend on density, the increase in divergence degrades the brilliance. The brilliance at $10 \mathrm{keV}$ thus reaches the maximum $B=5.6 \times$ $10^{20}$ photons $/ \mathrm{s} / \mathrm{mm}^{2} / \mathrm{mrad}^{2} / 0.1 \% \mathrm{BW}$ for the optimal density $n_{e}=1.1 \times 10^{18} \mathrm{~cm}^{-3}$.

Emitted radiation has also been calculated for the blowout regime (Sec. III). As we are much closer to a monoenergetic electron distribution, the result has been fitted with a synchrotron spectrum given by the formula $\frac{d I}{d \omega}=\frac{\omega}{\omega_{c}} \int_{\omega / \omega_{c}}^{\infty} K_{5 / 3}(\xi) d \xi$ where $K_{5 / 3}$ is the modified Bessel
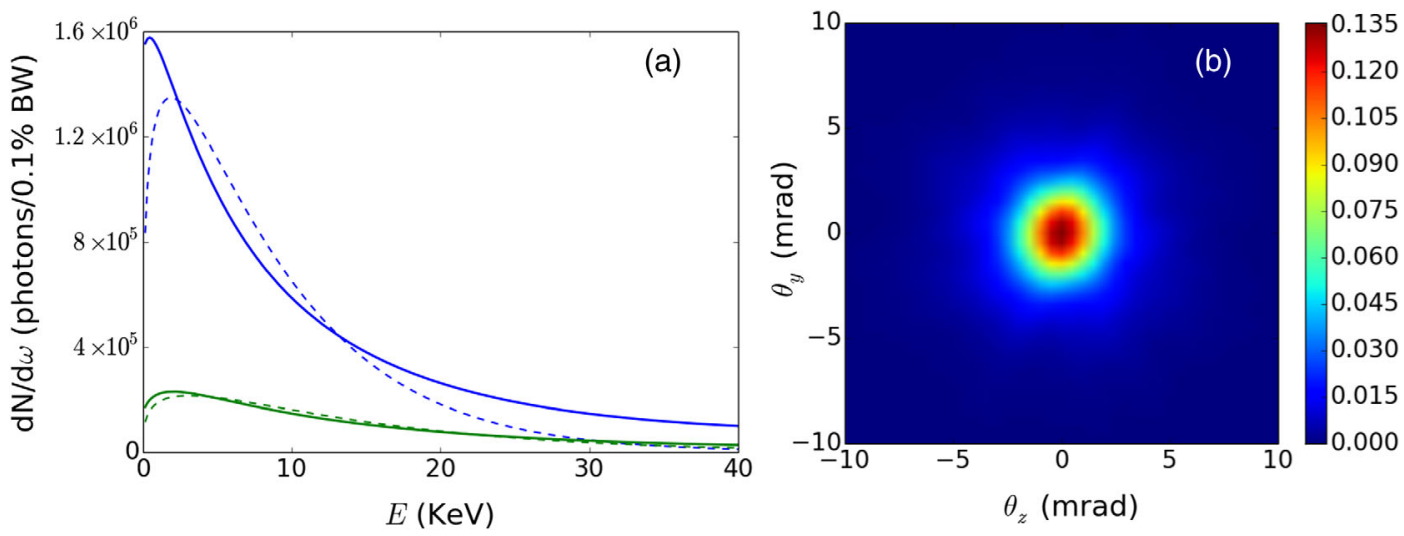

FIG. 9. Characteristics of x-ray emission in the blowout regime after $9 \mathrm{~cm}$ of propagation. (a) Solid curves: number of photons per $0.1 \% \mathrm{BW}$ emitted through the entire simulation (blue) and only over the last centimeter of propagation (green). Dashed curves: synchrotron fits. (b) Angular energy distribution $\frac{d I}{d \Omega}(\mathrm{J} / \mathrm{sr})$. 
function of the second kind with $\omega_{c}$ the critical frequency of the synchrotron spectrum. The radiation emitted during the entire simulation can be compared with a synchrotron spectrum with a critical energy $E_{c}=\hbar \omega_{c}=6.6 \mathrm{keV}$ [Fig. 9(a)]. This theoretical fit poorly matches the simulation results due to continuous electron acceleration. Conversely, radiation emitted during the last centimeter of propagation has a spectrum that is well approximated by a theoretical synchrotron spectrum with $E_{c}=10.9 \mathrm{keV}$. For an electron energy of $1.6 \mathrm{GeV}$, this corresponds to the theoretical critical frequency expected for $r_{\beta}=7.6 \mu \mathrm{m}$, which is quite close to the transverse size of the electron bunch measured in the simulation (cf. Table I). This can be compared to a critical energy of $E_{c}=26 \mathrm{keV}$ for the simulation with $n_{e}=$ $1.1 \times 10^{18} \mathrm{~cm}^{-3}$ in the SM-LWFA regime calculated by the same method, even though the procedure is not quite rigorous: given the Maxwellian electron distribution, the synchrotron fit strongly underestimates the number of photons emitted at high energy.

The lower injected charge and plasma density in the blowout regime reduce the photon number by a factor 10-400 against the SM-LWFA regime (see Table I). On the other hand, the blowout regime produces a much smaller source size ( $7 \mu \mathrm{m}$ against $25 \mu \mathrm{m}$ in the SM-LWFA regime) and duration (down by one order of magnitude from $250-300 \mathrm{fs}$ to $20 \mathrm{fs}$ ). The accelerated electron beam is much shorter as it fits into the rear of the leading electron cavity, rather than spreads over many wake buckets. More compact source favors $\mathrm{x}$-ray imaging with a high temporal and spatial resolution. Moreover, as the x-ray divergence is also reduced in the blowout regime, the brilliance is increased by a factor 60 against the best conditions under the SM-LWFA regime.

\section{SUMMARY AND OUTLOOK}

In this paper, we explore the prospects of using $\mathrm{kJ}$-energy, subpicosecond laser pulses for electron acceleration and production of pulsed synchrotron $\mathrm{x}$-rays in low-density plasmas. The regimes with the pulse longitudinal breakup (self-modulation) and electron blowout are explored. The advantage of the first approach is its simplicity. Self-modulation warrants massive electron injection (without tailoring the target) and acceleration of quasithermal electrons to a few $\mathrm{GeV}$ energy (with a $\mathrm{nC}$ charge above $1 \mathrm{GeV}$ ). Besides, the $\mathrm{x}$-ray source dimensions ( $\sim 25 \mu \mathrm{m}$ transverse size, $\sim 250$ fs duration) are almost insensitive to the background plasma density. This regime leads to highly charged electron bunches with quasiMaxwellian spectra. The x-ray beams have a high number of photons (up to $\sim 8 \times 10^{11}$ ) with the energies in tens of $\mathrm{keVs}$ and a relatively large divergence $(\approx 50 \mathrm{mrad}$ in the optimal case). Conversely, the blowout regime features a background-free, quasimonoenergetic electron distribution, with a sub-10\% energy spread around the central energy
1.6 GeV. Much lower charge $(\approx 1 \mathrm{nC})$ and tighter collimation of electron beam result in a modest photon yield $\left(2 \times 10^{9}\right.$ photons $)$ and lower photon energies. Yet, this regime yields a much more compact source $(7 \mu \mathrm{m}$ transverse size, 20 fs duration) and has a 260 times higher brilliance than in the best case of SM-LWFA.

Pursuit of further improvement in X-ray source characteristics may follow several routes. Operation in the blowout regime at higher densities (up to $n_{e} \approx 7 \times 10^{16} \mathrm{~cm}^{-3}$ ), provided the laser beam quality is sufficiently high to avoid disruptive filamentation, should enable longer selfguiding, thus increasing electron energy and the x-ray source energy and brightness. Alternatively, using a plasma channel at low densities [65] $\left(n_{e}<3 \times 10^{16} \mathrm{~cm}^{-3}\right)$ should prevent premature diffraction of the pulse and help maintain acceleration through dephasing. For controlling the injection, plasma lens could be a viable option as it essentially uses the same experimental design as the downramp injector $[43,66]$. Furthermore, filling a first injection-stage with helium doped with a high- $Z$ gas (e.g., nitrogen) may enable ionization-induced injection over a limited time, leading to the generation of quasimonoenergetic beams $[67,68]$. These options are the subject of further investigation. We conclude that petawatt, picosecond laser facilities, such as PETAL at LMJ $[38,41]$ and the Advanced Radiographic Capability (ARC) at NIF [39], may effectively drive high-brilliance, pulsed synchrotron x-rays, providing an invaluable option of ultrafast, high-resolution imaging of high energy density plasmas.

\section{ACKNOWLEDGMENTS}

This project was sponsored by Laserlab-Europe, EC-GA 284464. Work of S. Y. K. has been supported by the National Science Foundation Grant No. PHY-1535678.

[1] E. Esarey, C. B. Schroeder, and W. P. Leemans, Physics of laser-driven plasma-based electron accelerators, Rev. Mod. Phys. 81, 1229 (2009).

[2] V. Malka, Laser plasma accelerators, Phys. Plasmas 19, 055501 (2012).

[3] T. Tajima and J. M. Dawson, Laser Electron Accelerator, Phys. Rev. Lett. 43, 267 (1979).

[4] J. Faure, Y. Glinec, A. Pukhov, S. Kiselev, S. Gordienko, E. Lefebvre, J. P. Rousseau, F. Burgy, and V. Malka, A laserplasma accelerator producing monoenergetic electron beams, Nature (London) 431, 541 (2004).

[5] C. G. R. Geddes, Cs. Toth, J. van Tilborg, E. Esarey, C. B. Schroeder, D. Bruhwiler, C. Nieter, J. Cary, and W. P. Leemans, High-quality electron beams from a laser wakefield accelerator using plasma-channel guiding, Nature (London) 431, 538 (2004).

[6] S. P. D. Mangles, C. D. Murphy, Z. Najmudin, A. G. R. Thomas, J. L. Collier, A. E. Dangor, E. J. Divall, P. S. Foster, J. G. Gallacher, C. J. Hooker, D. A. Jaroszynski, 
A. J. Langley, W. B. Mori, P. A. Norreys, F. S. Tsung, R. Viskup, B. R. Walton, and K. Krushelnick, Monoenergetic beams of relativistic electrons from intense laser-plasma interactions, Nature (London) 431, 535 (2004).

[7] D. A. Edwards and M. J. Syphers, An Introduction to the Physics of High Energy Accelerators (WileyVCH, Weinheim, 2004).

[8] K. Schmid, L. Veisz, F. Tavella, S. Benavides, R. Tautz, D. Herrmann, A. Buck, B. Hidding, A. Marcinkevicius, U. Schramm, M. Geissler, J. Meyer-ter-Vehn, D. Habs, and F. Krausz, Few-Cycle Laser-Driven Electron Acceleration, Phys. Rev. Lett. 102, 124801 (2009).

[9] Z.-H. He, B. Hou, V. Lebailly, J. Nees, K. Krushelnick, and A. Thomas, Coherent control of plasma dynamics, Nat. Commun. 6, 7156 (2015).

[10] X. Wang et al., Quasi-monoenergetic laser-plasma acceleration of electrons to $2 \mathrm{GeV}$, Nat. Commun. 4, 1988 (2013).

[11] W. P. Leemans, B. Nagler, A. J. Gonsalves, Cs. Toth, K. Nakamura, C. G. R. Geddes, E. Esarey, C. B. Schroeder, and S. M. Hooker, GeV electron beams from a centimetrescale accelerator, Nat. Phys. 2, 696 (2006).

[12] S. Banerjee, N.D. Powers, V. Ramanathan, I. Ghebregziabher, K. J. Brown, C. M. Maharjan, S. Chen, A. Beck, E. Lefebvre, S. Y. Kalmykov, B. A. Shadwick, and D. P. Umstadter, Generation of tunable, 100-800 MeV quasi-monoenergetic electron beams from a laser-wakefield accelerator in the blowout regime, Phys. Plasmas 19, 056703 (2012).

[13] S. Banerjee, S. Y. Kalmykov, N. D. Powers, G. Golovin, V. Ramanathan, N. J. Cunningham, K. J. Brown, S. Chen, I. Ghebregziabher, B. A. Shadwick, D. P. Umstadter, B. M. Cowan, D. L. Bruhwiler, A. Beck, and E. Lefebvre, Stable, tunable, quasimonoenergetic electron beams produced in a laser wakefield near the threshold for self-injection, Phys. Rev. ST Accel. Beams 16, 031302 (2013).

[14] H. T. Kim, K. H. Pae, H. J. Cha, I. J. Kim, T. J. Yu, J. H. Sung, S. K. Lee, T. M. Jeong, and J. Lee, Enhancement of Electron Energy to the Multi-GeV Regime by a Dual-Stage Laser-Wakefield Accelerator Pumped by Petawatt Laser Pulses, Phys. Rev. Lett. 111, 165002 (2013).

[15] W. P. Leemans, A. J. Gonsalves, H.-S. Mao, K. Nakamura, C. Benedetti, C. B. Schroeder, Cs. Toth, J. Daniels, D. E. Mittelberger, S. S. Bulanov, J.-L. Vay, C. G. R. Geddes, and E. Esarey, Multi-GeV Electron Beams from CapillaryDischarge-Guided Subpetawatt Laser Pulses in the SelfTrapping Regime, Phys. Rev. Lett. 113, 245002 (2014).

[16] S. P. D. Mangles, B. R. Walton, M. Tzoufras, Z. Najmudin, R. J. Clarke, A. E. Dangor, R. G. Evans, S. Fritzler, A. Gopal, C. Hernandez-Gomez, W. B. Mori, W. Rozmus, M. Tatarakis, A. G. R. Thomas, F. S. Tsung, M. S. Wei, and K. Krushelnick, Electron Acceleration in Cavitated Channels Formed by a Petawatt Laser in Low-Density Plasma, Phys. Rev. Lett. 94, 245001 (2005).

[17] C. E. Clayton, J. E. Ralph, F. Albert, R. A. Fonseca, S. H. Glenzer, C. Joshi, W. Lu, K. A. Marsh, S. F. Martins, W. B. Mori, A. Pak, F. S. Tsung, B. B. Pollock, J. S. Ross, L. O. Silva, and D. H. Froula, Self-Guided Laser Wakefield Acceleration beyond $1 \mathrm{GeV}$ Using Ionization-Induced Injection, Phys. Rev. Lett. 105, 105003 (2010).
[18] H. Lu, M. Liu, W. Wang, C. Wang, J. Liu, A. Deng, J. Xu, C. Xia, W. Li, H. Zhang, X. Lu, C. Wang, J. Wang, X. Liang, Y. Leng, B. Shen, K. Nakajima, R. Li, and Z. Xu, Laser wakefield acceleration of electron beams beyond $1 \mathrm{GeV}$ from an ablative capillary discharge waveguide, Appl. Phys. Lett. 99, 091502 (2011).

[19] V. Ramanathan, S. Banerjee, N. Powers, N. Cunningham, N. A. Chandler-Smith, K. Zhao, K. Brown, D. Umstadter, S. Clarke, S. Pozzi, J. Beene, C. R. Vane, and D. Schultz, Submillimeter-resolution radiography of shielded structures with laser-accelerated electron beams, Phys. Rev. ST Accel. Beams 13, 104701 (2010).

[20] V. Malka, J. Faure, Y. Glinec, and Y.-A. Gauduel, Les sources de particules ultra-brèves, L'actualité Chimique 292, 18 (2005).

[21] L. Laschinsky, M. Baumann, E. Beyreuther, W. Enghardt, M. Kaluza, L. Karsch, E. Lessmann, D. Naumburger, M. Nicolai, C. Richter, R. Sauerbrey, H.-P. Schlenvoigt, and J. Pawelke, Radiobiological effectiveness of laser accelerated electrons in comparison to electron beams from a conventional linear accelerator, J. Radiat. Res. 53, 395 (2012).

[22] N. J. Cunningham, S. Banerjee, V. Ramanathan, N. Powers, N. Chandler-Smith, R. Vane, D. Schultz, S. Pozzi, S. Clarke, J. Beene, and D. P. Umstadter, High-energy laser-accelerated electron beams for long-range interrogation, AIP Conf. Proc. 1099, 638 (2009).

[23] S. Corde, K. Ta Phuoc, G. Lambert, R. Fitour, V. Malka, A. Rousse, A. Beck, and E. Lefebvre, Femtosecond x rays from laser-plasma accelerators, Rev. Mod. Phys. 85, 1 (2013).

[24] S. Kiselev, A. Pukhov, and I. Kostyukov, X-ray Generation in Strongly Nonlinear Plasma Waves, Phys. Rev. Lett. 93, 135004 (2004).

[25] A. Rousse, K. T. Phuoc, R. Shah, A. Pukhov, E. Lefebvre, V. Malka, S. Kiselev, F. Burgy, J.-P. Rousseau, D. Umstadter, and D. Hulin, Production of a keV X-Ray Beam from Synchrotron Radiation in Relativistic LaserPlasma Interaction, Phys. Rev. Lett. 93, 135005 (2004).

[26] S. Kneip et al., Bright spatially coherent synchrotron X-rays from a table-top source, Nat. Phys. 6, 980 (2010).

[27] S. Cipiccia et al., Gamma-rays from harmonically resonant betatron oscillations in a plasma wake, Nat. Phys. 7, 867 (2011).

[28] J. Ju and B. Cros, Characterization of temporal and spatial distribution of hydrogen gas density in capillary tubes for laser-plasma experiments, J. Appl. Phys. 112, 113102 (2012).

[29] J. Ju, K. Svensson, H. Ferrari, A. Dpp, G. Genoud, F. Wojda, M. Burza, A. Persson, O. Lundh, C.-G. Wahlstrm, and B. Cros, Study of electron acceleration and x-ray radiation as a function of plasma density in capillaryguided laser wakefield accelerators, Phys. Plasmas 20, 083106 (2013).

[30] J. Ju, G. Genoud, H. E. Ferrari, O. Dadoun, B. Paradkar, K. Svensson, F. Wojda, M. Burza, A. Persson, O. Lundh, N. E. Andreev, C.-G. Wahlström, and B. Cros, Analysis of x-ray emission and electron dynamics in a capillary-guided laser wakefield accelerator, Phys. Rev. ST Accel. Beams 17, 051302 (2014). 
[31] S. Fourmaux, S. Corde, K. Ta Phuoc, P. Lassonde, G. Lebrun, S. Payeur, F. Martin, S. Sebban, V. Malka, A. Rousse, and J.C. Kieffer, Single shot phase contrast imaging using laser-produced Betatron $\mathrm{X}$-ray beams, Opt. Lett. 36, 2426 (2011).

[32] S. Kneip, C. McGuffey, F. Dollar, M. S. Bloom, V. Chvykov, G. Kalintchenko, K. Krushelnick, A. Maksimchuk, S. P. D. Mangles, T. Matsuoka, Z. Najmudin, C. A. J. Palmer, J. Schreiber, W. Schumaker, A. G. R. Thomas, and V. Yanovsky, X-ray phase contrast imaging of biological specimens with femtosecond pulses of betatron radiation from a compact laser plasma wakefield accelerator, Appl. Phys. Lett. 99, 093701 (2011).

[33] Z. Najmudin, S. Kneip, M. Bloom, S. Mangles, O. Chekhlov, A. Dangor, A. Döpp, K. Ertel, S. Hawkes, J. Holloway, C. J. Hooker, J. Jiang, N. C. Lopes, H. Nakamura, P. A. Norreys, P. P. Rajeev, C. Russo, M. J. V. Streeter, D. R. Symes, and M. Wing, Compact laser accelerators for X-ray phase-contrast imaging, Phil. Trans. R. Soc. A 372, 20130032 (2014).

[34] S. Kneip, S. R. Nagel, C. Bellei, N. Bourgeois, A. E. Dangor, A. Gopal, R. Heathcote, S. P. D. Mangles, J. R. Marquès, A. Maksimchuk, P. M. Nilson, K. T. Phuoc, S. Reed, M. Tzoufras, F. S. Tsung, L. Willingale, W. B. Mori, A. Rousse, K. Krushelnick, and Z. Najmudin, Observation of Synchrotron Radiation from Electrons Accelerated in a Petawatt-Laser-Generated Plasma Cavity, Phys. Rev. Lett. 100, 105006 (2008).

[35] D. Maywar et al., OMEGA EP high-energy petawatt laser: Progress and prospects, J. Phys. Conf. Ser. 112, 032007 (2008).

[36] N. Miyanaga, H. Azechi, K. Tanaka, T. Kanabe, T. Jitsuno, J. Kawanaka, Y. Fujimoto, R. Kodama, H. Shiraga, K. Knodo, K. Tsubakimoto, H. Habara, J. Lu, G. Xu, N. Morio, S. Matsuo, E. Miyaji, Y. Kawakami, Y. Izawa, and K. Mima, 10-kJ PW laser for the FIREX-I program, J. Phys. IV 133, 81 (2006).

[37] V. Bagnoud et al., Commissioning and early experiments of the PHELIX facility, Appl. Phys. B 100, 137 (2010).

[38] N. Blanchot et al., Overview of PETAL, the multi-Petawatt project in the LMJ facility, Eur. Phys. J. Web Conf. 59, 07001 (2013).

[39] C. Barty et al., An overview of LLNL high-energy shortpulse technology for advanced radiography of laser fusion experiments, Nucl. Fusion 44, S266 (2004).

[40] S. H. Glenzer and R. Redmer, X-ray Thomson scattering in high energy density plasmas, Rev. Mod. Phys. 81, 1625 (2009).

[41] D. Batani et al., Development of the PETAL laser facility and its diagnostic tools, Acta Polytechnica 53, 103 (2013).

[42] S. Y. Kalmykov et al., Numerical modelling of a 10-cmlong multi-GeV laser wakefield accelerator driven by a self-guided petawatt pulse, New J. Phys. 12, 045019 (2010).

[43] S. Y. Kalmykov, S. A. Yi, A. Beck, A. F. Lifschitz, X. Davoine, E. Lefebvre, V. Khudik, G. Shvets, and M. C. Downer, Dark-current-free petawatt laser-driven wakefield accelerator based on electron self-injection into an expanding plasma bubble, Plasma Phys. Controlled Fusion 53, 014006 (2011).
[44] S. Y. Kalmykov, Dark-current-free laser-plasma acceleration in blowout regime using nonlinear plasma lens, AIP Conf. Proc. 1507, 921 (2012).

[45] J. B. Rosenzweig, B. Breizman, T. Katsouleas, and J. J. Su, Acceleration and focusing of electrons in two-dimensional nonlinear plasma wake fields, Phys. Rev. A 44, R6189 (1991).

[46] P. Mora and T. M. Antonsen Jr., Electron cavitation and acceleration in the wake of an ultraintense, self-focused laser pulse, Phys. Rev. E 53, R2068 (1996).

[47] A. Pukhov and J. Meyer-ter Vehn, Laser wake field acceleration: the highly non-linear broken-wave regime, Appl. Phys. B 74, 355 (2002).

[48] W. Lu, C. Huang, M. Zhou, M. Tzoufras, F. Tsung, W. Mori, and T. Katsouleas, A nonlinear theory for multidimensional relativistic plasma wave wakefields, Phys. Plasmas 13, 056709 (2006).

[49] S. Y. Kalmykov, A. Beck, S. A. Yi, V. N. Khudik, M. C. Downer, E. Lefebvre, B. A. Shadwick, and D.P. Umstadter, Electron self-injection into an evolving plasma bubble: Quasi-monoenergetic laser-plasma acceleration in the blowout regime, Phys. Plasmas 18, 056704 (2011).

[50] S. Gordienko and A. Pukhov, Scalings for ultrarelativistic laser plasmas and quasimonoenergetic electrons, Phys. Plasmas 12, 043109 (2005).

[51] W. Lu, M. Tzoufras, C. Joshi, F. S. Tsung, W. B. Mori, J. Vieira, R. A. Fonseca, and L. O. Silva, Generating multi$\mathrm{GeV}$ electron bunches using single stage laser wakefield acceleration in a 3D nonlinear regime, Phys. Rev. ST Accel. Beams 10, 061301 (2007).

[52] G.-Z. Sun, E. Ott, Y. Lee, and P. Guzdar, Self-focusing of short intense pulses in plasmas, Phys. Fluids 30, 526 (1987).

[53] C. Decker, W. Mori, K.-C. Tzeng, and T. Katsouleas, The evolution of ultra-intense, short-pulse lasers in underdense plasmas, Phys. Plasmas 3, 2047 (1996).

[54] P. Gibbon, The self-trapping of light waves by beat-wave excitation, Phys. Fluids B 2, 2196 (1990).

[55] N. E. Andreev, L. M. Gorbunov, V. I. Kirsanov, A. A. Pogosova, and R. R. Ramazashvili, Self-resonant wakefield generation by intense laser pulse in plasmas, Proc. SPIE Int. Soc. Opt. Eng. 1928, 39 (1993).

[56] P. Sprangle, E. Esarey, J. Krall, and G. Joyce, Propagation and guiding of intense laser pulses in plasmas, Phys. Rev. Lett. 69, 2200 (1992).

[57] T. M. Antonsen Jr. and P. Mora, Self-Focusing and Raman Scattering of Laser Pulses in Tenuous Plasmas, Phys. Rev. Lett. 69, 2204 (1992).

[58] B. Hidding, M. Geissler, G. Pretzler, K.-U. Amthor, H. Schwoerer, S. Karsch, L. Veisz, K. Schmid, and R. Sauerbrey, Quasimonoenergetic electron acceleration in the self-modulated laser wakefield regime, Phys. Plasmas 16, 043105 (2009).

[59] N. Lemos, J. L. Martins, F. S. Tsung, J. L. Shaw, K. A. Marsh, F. Albert, B. B. Pollock, and C. Joshi, Selfmodulated laser wakefield accelerators as X-ray sources, Plasma Phys. Controlled Fusion 58, 034018 (2016).

[60] A. F. Lifschitz, X. Davoine, E. Lefebvre, J. Faure, C. Rechatin, and V. Malka, Particle-in-Cell modelling of 
laser-plasma interaction using Fourier decomposition, J. Comput. Phys. 228, 1803 (2009).

[61] C. G. R. Geddes, K. Nakamura, G. R. Plateau, C. Toth, E. Cormier-Michel, E. Esarey, C. B. Schroeder, J. R. Cary, and W. P. Leemans, Plasma-Density-Gradient Injection of Low Absolute-Momentum-Spread Electron Bunches, Phys. Rev. Lett. 100, 215004 (2008).

[62] S. A. Yi, V. Khudik, C. Siemon, and G. Shvets, Analytic model of electromagnetic fields around a plasma bubble in the blow-out regime, Phys. Plasmas 20, 013108 (2013).

[63] P. Mora and T.M. Antonsen, Jr., Kinetic modeling of intense, short laser pulses propagating in tenuous plasmas, Phys. Plasmas 4, 217 (1997).

[64] J. D. Jackson, Classical Electrodynamics (Wiley, New York, 1975).

[65] N. A. Bobrova, S. V. Bulanov, A. A. Esaulov, and P. V. Sasorov, Capillary discharges for guiding of laser pulses, Plasma Phys. Rep. 26, 10 (2000).
[66] A. Gonsalves, K. Nakamura, C. Lin, D. Panasenko, S. Shiraishi, T. Sokollik, C. Benedetti, C. Schroeder, C. Geddes, J. van Tilborg, J. Osterhoff, E. Esarey, C. Toth, and W. P. Leemans, Tunable laser plasma accelerator based on longitudinal density tailoring, Nat. Phys. 7, 862 (2011).

[67] B. B. Pollock, C. E. Clayton, J. E. Ralph, F. Albert, A. Davidson, L. Divol, C. Filip, S. H. Glenzer, K. Herpoldt, W. Lu, K. A. Marsh, J. Meinecke, W. B. Mori, A. Pak, T. C. Rensink, J. S. Ross, J. Shaw, G. R. Tynan, C. Joshi, and D. H. Froula, Demonstration of a Narrow Energy Spread, $\sim 0.5 \mathrm{GeV}$ Electron Beam from a Two-Stage Laser Wakefield Accelerator, Phys. Rev. Lett. 107, 045001 (2011).

[68] G. Golovin, S. Chen, N. Powers, C. Liu, S. Banerjee, J. Zhang, M. Zeng, Z. Sheng, and D. Umstadter, Tunable monoenergetic electron beams from independently controllable laser-wakefield acceleration and injection, Phys. Rev. ST Accel. Beams 18, 011301 (2015). 